\title{
EFFECT OF SOLVENT POLARITY ON THE EFFICIENCY OF ULTRASOUND-ASSISTED EXTRACTION OF POLYPHENOLS FROM APPLE POMACE
}

\author{
Zbigniew KOBUS, Kamil WILCZYŃSKI, Rafał NADULSKI, Leszek RYDZAK, \\ Tomasz GUZ \\ University of Life Sciences in Lublin, POLAND \\ E-mail of corresponding author: zbigniew.kobus@up.lublin.pl
}

Keywords: apple pomace, ultrasound, polyphenols, energy consumption, extraction efficiency

\begin{abstract}
In this paper influence of solvent polarity on the efficiency of ultrasound-assisted extraction of polyphenols from apple pomace was studied. In the experiment, three solvents were used: water, $60 \%$ ethanol and $96 \%$ ethanol. The extraction process was aided by ultrasonic waves at intensity of $60 \mathrm{~W}$. Efficiency of extraction was determined by measuring total phenolic content, antioxidant activity, energy consumption and unit energy consumption. It has been shown that a $60 \%$ solution of ethanol is the most effective solvent for ultrasound-assisted extraction of polyphenols from apple pomace. The application of an appropriate solvent allows a significant reduction in the energy consumption of the extraction process, what is important for the sustainable use of energy sources in the food and agriculture sector.
\end{abstract}

\section{INTRODUCTION}

Waste utilization is a very important issue in the development of sustainable agriculture. Apple pomace is the main by-product of the industrial production of apple juice and cider (Reis et al., 2012). The basic way of pomace utilization is use of it in cattle feed. Apple pomace can be also applied as fuel. Because of its high carbon and nitrogen content, up to $80 \%$ of organic matter the pomace can be converted into high-energy gas. The better way of pomace using is production of food preparations. The high content of fiber causes the waste bond metals well, what improves the removing it's from organism. On the other hand high content of polyphenols makes it possible to use the pomace for the production of preparations with increased antioxidant activity (Cao et al., 2009; Ćetković et al., 2008).

Extraction is one of method for production of phenol preparations. The convectional extraction techniques such as maceration, percolation, extraction with mixing or Soxhlet extraction are usually time-consuming. Because of the above, new methods are being searched to increase the yield of extraction and to provide high quality of antioxidant components at the same time (Chemat et al., 2012). There are different methods of extraction aided: pulsed electric field (Lohani and Muthukumarappan 2016; Schillinga et al., 2007), microwave (Grigoras et al., 2013) or ultrasound (Kobus 2008).

The ultrasonic assisted extraction method significantly shortens the time of the process and increases the extraction of phenolic compounds from fruit waste including apple pomace (Pradal et al., 2016). The ultrasonic extraction mechanism itself is widely known, but still different research is conducted into factors affecting the process efficiency such as: frequency and intensity of ultrasound, time and temperature of the process and nature of the solvent (Kobus and Kusińska 2008).

The selection of a suitable solvent during ultrasonic extraction has a significant effect on the content of polyphenol compounds obtained from apple pomace and reduces the energy consumption (Pradal et al., 2016). The polar nature of the solvent affects the content of substances extracted from the solid and passing to the solvent. From the 
eluotropic solvent series only ethanol and water are used in the food industry because of the non-toxic nature. Between these solvents, a large difference in the Snyder polarity index can be observed, respectively: 5.2 for ethanol and 9.0 for water (Snyder 1974). In both solvents, there is a hydroxyl group, which makes it possible to form hydrogen bonds with soluble substances. Available results confirm that the effectivity of polyphenols extraction depends on the chemical structure of the extractant and the propagation of ultrasonic waves in liquid media.

The purpose of the study was to investigate the effect of solvent polarity on the process of ultrasonic extraction of polyphenols from apple pomace.

\section{MATERIAL AND METHODS}

The material used for the study was the apple pomace of the Jonagold variety bought at the local supermarket Tesco (Lublin, Poland). The apples after the wash were crushed and the juice was centrifuged in the juicer. Three types of solvents were used: water, ethanol $96 \%$ and ethanol $60 \%$. A sample of $20 \mathrm{~g}$ of the pomace was mixed with $200 \mathrm{ml}$ of solvent. Ultrasound-assisted extraction was performed using an ultrasonic processor (Sonic VCX 750) with a frequency of $20 \mathrm{kHz}$. The ultrasonic intensity was $60 \mathrm{~W}$ and the processing time $30 \mathrm{~min}$. The extraction process was carried out in a water bath (Brookfield TC-502) maintaining a constant temperature of $25^{\circ} \mathrm{C}$.

The content of polyphenols was measured using a spectrophotometer (Shimadzu UV1800, Japan) using a modified Singleton method based on Folin-Ciocalteu reagent at 760 $\mathrm{nm}$ (Singleton et al., 1965). The total polyphenol content was expressed as mg gallic acid equivalent per 100 grams of apple pomace.

Antioxidant activity was determined according to the modified Brand-Wiliam method and co-workers using the DPPH (1,1-diphenyl-2-picrylhydrazyl) radical (BrandWilliams et al., 1995). For the analysis, $0.2 \mathrm{ml}$ of apple juice was mixed with freshly prepared solution of DPPH radical dissolved in methanol. After 30 minutes of incubation in a dark room, the absorbance of the solution was measured at $516 \mathrm{~nm}$. The antioxidant activity was expressed as the percentage inhibition of the DPPH radical calculated from the formula:

$$
\mathrm{AA}(\%)=\frac{\mathrm{A}_{0}-\mathrm{A}_{\mathrm{t}}}{\mathrm{A}_{0}} \cdot 100 \%
$$

where:

$\mathrm{A}_{0}$ - initial DPPH absorbance value,

$A_{t}-$ absorbance of the DPPH radical after a fixed reaction time.

The analysis energy consumption of ultrasonic-assisted extraction was performed with respect to the polyphenol contents obtained in the apple pomace. The unit energy input was determined by the following formula:

$$
N=\frac{E}{m}
$$

where:

E - energy consumption $(\mathrm{kJ})$,

$\mathrm{m}$ - mass of polyphenols ( $\mathrm{g}$ ). 
Each measurement was done in triplicate. The results of the study were statistically analyzed in the Statistica program using the one-way analysis of variance ANOVA. The significance of differences between means using the t-Tukey test was also investigated.

\section{RESULTS}

The physical and chemical properties of solvent play an important role in extraction process. The solvent has a significant effect on the amount of substance eluted from the solid matrix. The properties of the solvent affect its applicability and the cost of the extraction process. Figure 1 shows the total polyphenol content for 3 extractant types expressed in $\mathrm{mg}$ gallic acid per $100 \mathrm{~g}$ apple pomace.

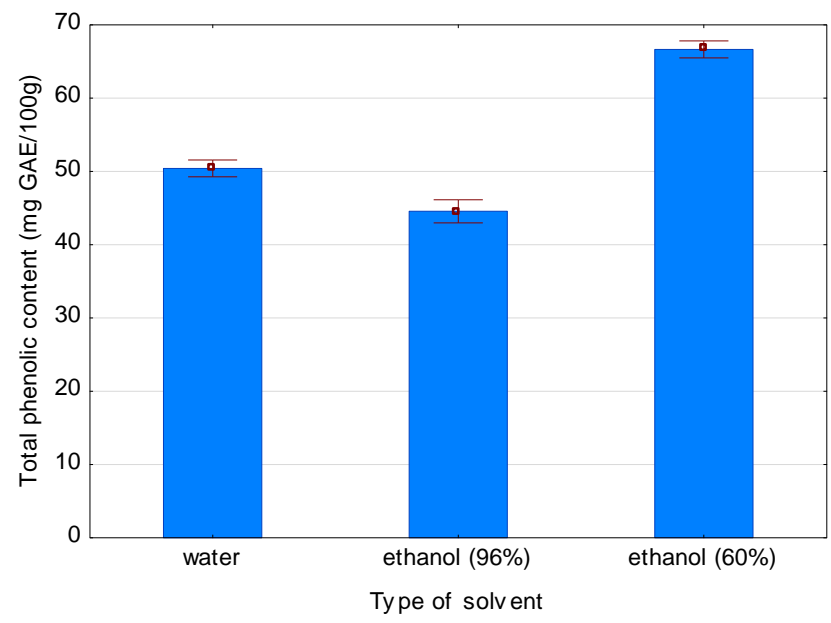

Fig. 1. Influence of solvent polarity on yield of ultrasound-assisted extraction

There were statistically significant differences between the total content of polyphenols and the type of solvent. The highest extraction yield was obtained with a mixture of water and ethanol (60\% ethanol) and the lowest with ethanol (96\%). The use of pure water as a solvent produced about $11 \%$ higher yield than $96 \%$ ethanol.

Water is more effective solvent due to higher polarity coefficient (Pin et al., 2009). The efficiency of the extraction process depends on the nature of the phenolic compounds present in the raw material. Gallic acid contains four hydroxyl groups and a carboxy group and is better soluble in highly polar solvents such as water.

Research conducted by Arize et al., (2014) also showed that water due to a higher polarity factor elutes more phenolic compounds from the solid than ethanol. Ethanol, due to its different molecular structure, is more effective in eluting such compounds as chlorogenic acid and flavonoids (Wijngaarda and Brunton 2010). Hence, a mixture of water and ethanol provides higher polyphenol extraction efficiency than the use of these solvents separately.

The important parameter determining the value of extracts is their ability to sweep free radicals (Panzella et al., 2013). The effect of solvent polarity on antioxidant activity is shown in Fig. 2. 


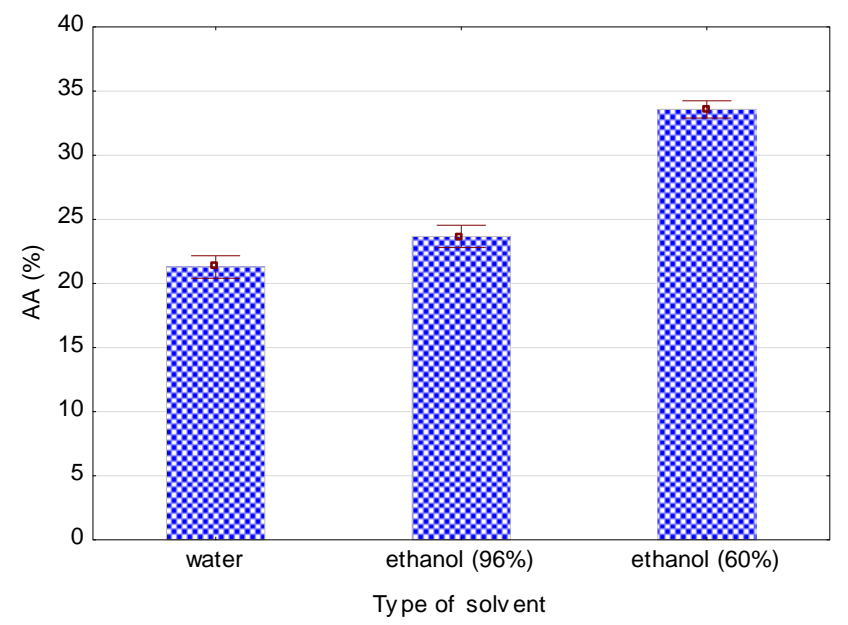

Fig. 2. Influence of solvent polarity on antioxidant activity (AA) in \% of obtaining extracts

There was a significant effect of solvent polarity on the antioxidant activity of the obtained extracts. The smallest value of this parameter was observed for the extract obtained with water, and the largest with a $60 \%$ solution of ethanol. Extracts obtained with a $96 \%$ ethanol solution had slightly higher antioxidant activity than aqueous extracts, despite the fact, that they contained less phenolic in total. These findings are consistent with the results obtained by other authors. Galván d'Alessandro et al., (2012) and Virot et al., (2010) studying the solubility of polyphenols in various mixtures of water and ethanol achieved the highest solubility of these compounds in a 50\% ethanol solution. Pradal et al., (2016) examining ultrasonic extraction of polyphenols from the root of chicory found the highest antioxidant activity in the case of $60 \%$ ethanol extracts. Wijngaard and Brunton (2010) extracting apple polyphenols (without the use of ultrasound) achieved the highest ability to sweep free radicals for extracts with an alcohol concentration from 50 to $60 \%$.

The changes of energy consumption in the ultrasonic extraction of polyphenol from apple pomace, depending on the type of solvent used, are shown in Figure 3.

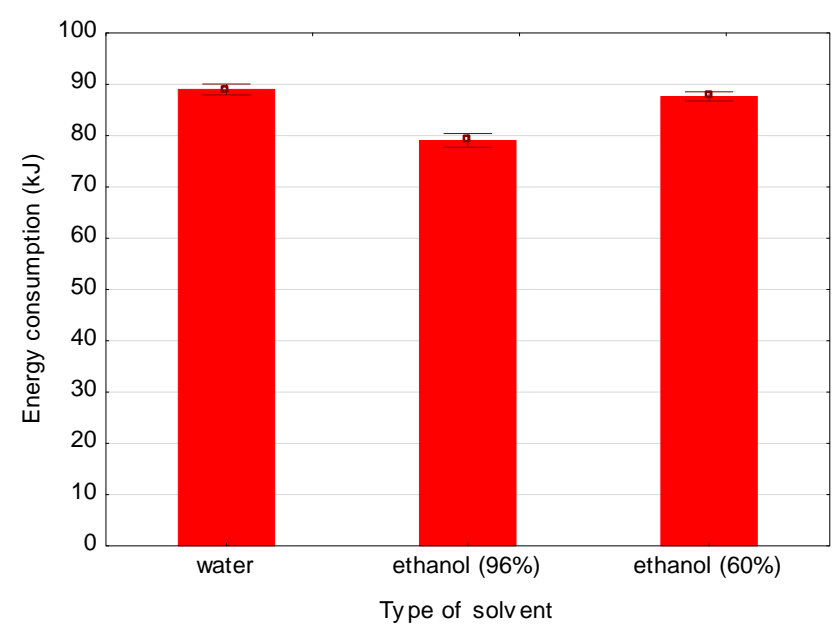

Fig. 3. Influence of solvent polarity on energy consumption (kJ) during ultrasound-assisted extraction

There was a statistically significant effect of the solvent type on the energy consumption during the ultrasonic extraction of polyphenols from apple pomace. The lowest energy 
consumption was observed during extraction with $96 \%$ ethanol and the lowest during water extraction.

Kobus and Kusińska (2008) showed that the type of solvent used and its physical properties have a significant effect on the amount of energy emitted by the ultrasonic head. This is because the constant amplitude of the ultrasound wave generated by the device depends on the resistance of the liquid. The ultrasonic processor consumes more energy in liquids with higher acoustic impedance. Hence, the energy generated by the sonicator during water extraction is higher than during the extraction with ethanol as a solvent.

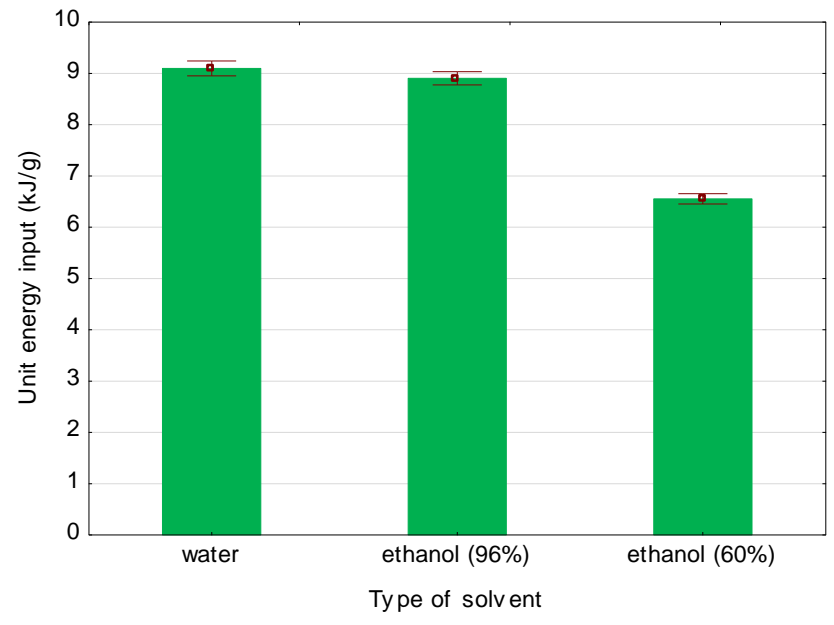

Fig. 4. Influence of solvent polarity on unit energy consumption $(\mathrm{kJ} / \mathrm{g})$ during ultrasound-assisted extraction

An important parameter during ultrasound-assisted extraction is the energy input needed to obtain the unit of extracted substance. Changes in this factor depending on the type of solvent are shown in Figure 4. There was a statistically significant effect of the type of solvent used on the value of unit energy input. The smallest unit energy input was observed during extraction with $60 \%$ water and ethyl alcohol, while the highest in extraction with pure water as a solvent. The results clearly show that during the ultrasound assisted extraction of polyphenols from apple pomace, the best solvent is a $60 \%$ mixture of ethyl alcohol and water.

\section{CONCLUSIONS}

In the paper, an evaluation of the type of solvent used on the efficiency of the ultrasound assisted extraction of polyphenols from apple pomace was carried out. The results showed that total polyphenol content ranged from 45 to $68 \mathrm{mg}$ GAE/100 g apple pomace, depending on the solvent used. The highest extraction yield was found in the case of $60 \%$ ethyl alcohol. Polyphenols obtained using this solvent also had the highest antioxidant activity. Energy efficiency analysis has shown that using $60 \%$ ethanol allows to obtain the lowest unit energy input. This input was about $28 \%$ lower than for the use of pure water as an extractant. Taking into account process efficiency and energy consumption, it is recommended to conduct the extraction of polyphenol from apple pomace using a $60 \%$ ethyl alcohol solution. The application of ultrasound-assisted extraction coupled with the selection of suitable solvent reduces energy consumption, what is important in the rational management of energy resources. 


\section{REFERENCES}

Azrie, A.M., Chuah, A.L., Pin, K.Y., Tan, H.P., (2014). Effect of solvents on the extraction of Kacip Fatimah (Labisia pumila) leaves. Journal of Chemical and Pharmaceutical Research, 6 (9), 172-176.

Brand-Williams, W., Cuvelier, M.E., Berset, C., (1995). Use of a free radical method to evaluate antioxidant activity. LWT-Food Science and Technology, 28 (1), 25-30.

Cao, X., Wang, C., Pei, H., Sun, B., (2009). Separation and identification of polyphenols in apple pomace by high-speed counter-current chromatography and highperformance liquid chromatography coupled with mass spectrometry. Journal of Chromatography A, 1216 (19), 4268-4274.

Ćetković, G., Čanadanović-Brunet, J., Djilas, S., Savatović, S., Mandić, A., Tumbas, V., (2008). Assessment of polyphenolic content and in vitro antiradical characteristics of apple pomace. Food Chemistry, 109 (2), 340-347.

Chemat, F., Vian, A., M., Cravotto, G., (2012). Green extraction of natural products: Concept and principles. International Journal of Molecular Sciences, 13, 8615-8627.

Galvan d'Alessandro, L., Kriaa, K., Nikov I., Dimitrov, K., (2012). Ultrasound assisted extraction of polyphenols from black chokeberry. Separation and Purification Technology, 93 (1), 42-47.

Grigoras, C.G., Destandaua, E., Fougèrea, L., Elfakira, C., (2013). Evaluation of apple pomace extracts as a source of bioactive compounds. Industrial Crops and Products, 49, 794-804.

Kobus Z., (2008). Dry matter extraction from valerian roots (Valeriana officinalis L.) with the help of pulsed acoustic field. International Agrophysics, 22, 133-137.

Kobus, Z., Kusińska, E., (2008). Influence of physical properties of liquid on acoustic power of ultrasonic processor. Teka Komis. Mot. Energ. Rol., 8, 71-78.

Lohani, U.C., Muthukumarappan, K., (2016). Application of the pulsed electric field to release bound phenolics in sorghum flour and apple pomace. Innovative Food Science and Emerging Technologies, 35, 29-35.

Panzella, L., Petriccione, M., Rega, P., Scortichini, M., Napolitano, A., (2013). A reappraisal of traditional apple cultivars from Southern Italy as a rich source of phenols with superior antioxidant activity. Food Chemistry, 140, 672-679.

Pin, K.Y., Chuah, T.G., Abdullah Rashih, A., Law, C.L., Rasadah, M.A., (2009). Drying of Betel Leaves (Piper betle L.): Quality and Drying Kinetics. Drying Technology, 27, 149-155.

Pradal, D., Vauchel, P., Decossin, S., Dhulster, P., Dimitrov, K., (2016). Kinetics of ultrasound-assisted extraction of antioxidant polyphenols from food by-products: Extraction and energy consumption optimization. Ultrasonics Sonochemistry, 32, 137-146.

Reis, S. F., Rai, D.K., Ghannam, N.A., (2012). Water at room temperature as a solvent for the extraction of apple pomace phenolic compounds. Food Chemistry, 135, 1991-1998.

Schillinga, S., Albera, T., Toepflb, S., Neidharta, S., Knorrb, D., Schiebera, A., (2007). Effects of pulsed electric field treatment of apple mash on juice yield and quality attributes of apple juices. Innovative Food Science and Emerging Technologies, 8 (1), 127-134.

Singleton, V.L., Rossi, J.A., (1965). Colorimetry of total phenolics with phosphomolybdicphosphotungstic acid reagents. American Journal of Enology Viticulture, 16 (3), 144-158.

Snyder, L.R., (1974). Classification of the solvent properties of common liquids. Journal of Chromatography A, 92, 233-240.

Virot, M., Tomao, V., Le Bourvellec, C., Renard C.M., Chemat, F., (2010). Towards the industrial production of antioxidants from food processing by-products with ultrasound-assisted extraction. Ultrasonics Sonochemistry, 17 (6), 1066-74.

Wijngaard, H.H., Brunton, N., (2010). The optimisation of solid-liquid extraction of antioxidants from apple pomace by response surface methodology. Journal of Food Engineering, 96 (1), 134-140. 